\title{
Downregulated microRNA-26a modulates prostate cancer cell proliferation and apoptosis by targeting COX-2
}

\author{
JING ZHANG $^{1^{*}}, \mathrm{JINGHAO} \mathrm{LIANG}^{2 *}$ and JIANGUO HUANG ${ }^{1}$ \\ Departments of ${ }^{1}$ Oncology and ${ }^{2}$ Orthopedics, \\ Urumqi General Hospital of Lanzhou Military Command of the Chinese People's Liberation Army, \\ Urumqi, Xinjiang 830000, P.R. China
}

Received May 27, 2015; Accepted May 24, 2016

DOI: $10.3892 / \mathrm{ol} .2016 .5070$

\begin{abstract}
MicroRNA-26a (miR-26a) is expressed at lower levels in prostate cancer cells compared with normal prostate cells. However, the regulatory mechanism of miR-26a in tumorigenesis and metastasis is not clear. In the present study, the expression profile of cellular miR-26a was analyzed by reverse transcription-quantitative polymerase chain reaction. The potential target of miR-26a was identified by luciferase assay and western blotting. Examination of miR-26a function was performed by transfection with miR-26a mimics and inhibitor. It was found that miR-26a expression was decreased in prostate cancer tissues and cell lines, with androgen-independent prostate cancer (AIPC) showing lower miR-26a expression compared with androgen-dependent prostate cancer (ADPC). Overexpression of miR-26a by transfecting miR-26a mimics could significantly enhance apoptosis, and this upregulation of apoptosis was triggered by cytochrome $c$ oxidase subunit II inhibition. Furthermore, it was found that lower miR-26a density resulted in an evidently poor prognosis. Understanding the important roles of miR-26a in regulating cell apoptosis in human prostate cancer cells may aid the exploration of AIPC transformation mechanisms and contribute to the development of miRNA-based therapy in the future.
\end{abstract}

\section{Introduction}

Prostate cancer is one of the most common malignant tumors in men and the mortality rate has reached the second highest ranking among all male cancers in the USA (1). Although the morbidity of prostate cancer in China is far

Correspondence to: Professor Jianguo Huang, Department of Oncology, Urumqi General Hospital of Lanzhou Military Command of the Chinese People's Liberation Army, 359 Youhao North Road, Urumqi, Xinjiang 830000, P.R. China

E-mail: hjgandhx@163.com

*Contributed equally

Key words: prostate cancer, apoptosis, microRNA, COX-2, AIPC lower than that of western countries, the morbidity has markedly increased in recent years, with an estimated incidence of 10 cases/100,000 individuals in 2010 compared with 1.71 cases/100,000 individuals in 1993 (2). Early-stage prostate cancer requires androgens for growth and thus responds well to androgen deprivation therapy (3). However, the majority of patients with androgen-dependent prostate cancer (ADPC) develop androgen-independent prostate cancer (AIPC) subsequent to the initiation of androgen deprivation. This indicates the next proliferation stage of prostate cancer cells following the remittent stage, such as continuous increase in prostate specific antigen (PSA) levels, and bone metastasis occurs extremely easily. At present, no curative therapy exists for this refractory disease.

The AIPC transformation mechanism is the focus of studies investigating prostate cancer. The primary reasons for ADPC becoming AIPC are gene expression variation, signal pathway abnormity, and dysregulation of proto-oncogenes, cancer suppressor genes and growth factors (4). At present, studies have identified a large number of relevant genes and signal pathways of prostate cancer (5-7). However, since the biological behavior of prostate cancer is extremely complex, any of the aforementioned hypotheses cannot clarify the pathogenetic mechanism of AIPC (6). Therefore, identification of genes involved in the transition from ADPC to AIPC is important to extend the current knowledge of AIPC (7).

The human transcriptome comprises not only large numbers of protein-coding messenger RNAs (mRNAs), but also a large set of non-protein coding transcripts that have structural, regulatory or unknown functions (8). Noncoding RNAs are divided into long noncoding RNAs (>200 nucleoides) and short noncoding RNAs [20-30 nucleotides; microRNAs (miRNAs)], according to their length. miRNAs are a class of small noncoding RNAs that are implicated in numerous physiological and pathological responses as post-transcriptional repressors of gene expression. Mature miRNAs can specifically bind to the 3'-untranslated regions (3'-UTRs) of target cellular mRNA, in turn triggering mRNA degradation or inhibition of translation (9). A previous study has reported that miRNA-26a (miR-26a) had decreased expression in prostate cancer (10). However, there is limited knowledge about the role of miR-26a in prostate cancer.

Increased cytochrome $c$ oxidase subunit II (COX-2) expression has been linked to the initiation and progression of human 
prostate cancer. TargetScan and luciferase activity analysis have confirmed COX-2 as a target of miR-26a (11), and COX-2 has been reported to play a key role in the apoptosis of cancer cells (12).

In the present study, it was found that miR-26a was significantly downregulated in prostate cancer tissues. Ectopic expression of miR-26a may increase cell proliferation and inhibit apoptosis. It was also found that miR-26a promotes apoptosis by targeting COX-2.

\section{Materials and methods}

Cell culture and cell transfection. The AIPC PC-3 cell line (purchased from the American Type Culture Collection, Manassas, VA, USA) were maintained in RPMI-1640 (Gibco; Thermo Fisher Scientific, Inc., Waltham, MA, USA) supplemented with $10 \%$ fetal bovine serum (Gibco; Thermo Fisher Scientific, Inc.), 2 mML-glutamine (Sangong, Shanghai, China) and $25 \mathrm{mM}$ HEPES (Sangong). miRNA mimics, inhibitors or scrambled miRNA-control were synthesized from GenePharma (Shanghai, China) products. pcDNA3.1-myc-COX-2 was purchased from Biogot Technology Co., Ltd. (Nanjing, China). COX-2 siRNA was obtained from RiboBio Co., Ltd. (Guangzhou, China) and the sequence was as follows: Forward, 5'-GCUGGGAAGCCUUCUCUAA-3'; and reverse, 5'-TCGACCCUUCGGAAGAGAUU-3'. Transfections were performed using Lipofectamine 2000 (Invitrogen; Thermo Fisher Scientific, Inc.) with miRNA mimics, inhibitors or scrambled control (RiboBio Co., Ltd.) for $24 \mathrm{~h}$.

Plasmid constructs and luciferase reporter assay. Targetscan was used to identify COX-2 as a potential miR-26a target (http://www.targetscan.org/). To construct the luciferase reporter vector, COX-2 3'-UTR and its flanking sequence was amplified by polymerase chain reaction (PCR) using the following primers: Forward, 5'-CGGGGTACCGAG TCATACTTGTGAAG-3'; and reverse, 5'-GCACTCGAG CCTGTTTTTGTTTGATG-3'. The amplified fragment was cloned into a PGL3 vector (Promega, Madison, WI, USA). Similarly, a mutated COX-2 3'-UTR fragment, in which the miR-26a binding site was mutated, was PCR-amplified using the following primers; Forward, 5'-GTGGTTTCAACTTAT ATTATAAGAACG-3'; and reverse, 5'-GACGAAAAGACG TCAAAACTCATTT-3'. The PCR product was cloned into the PGL3 vector. Luciferase activity assay was performed according to the manufacturer's instructions (Promega, Madison, WI, USA).

Clinical samples. All prostate cancer and adjacent normal tissues included in the present study were obtained from 32 patients with prostate adenocarcinoma that were diagnosed by two pathologists at the Department of Oncology, Urumqi General Hospital of Lanzhou Military Command of the Chinese People's Liberation Army (Urumqi, China). Medical history, transrectal ultrasound, computed tomography and magnetic resonance imaging findings, and isotope scanning of the skeleton were combined to decide the clinical staging. In total, 12 patients that underwent radical prostatectomy, did not have metastases and maintained extremely low PSA levels $(<0.2 \mathrm{ng} / \mathrm{ml})$, with no relapse, were diagnosed with ADPC, according to a previous study (13). In addition, 20 patients that presented with increases in PSA levels or bone metastases were determined to have advanced hormone-refractory prostate cancer. All experiments were performed according to the principles of the Helsinki Declaration. All patients enrolled in the present study provided informed consent. The study was conducted with the approval of the Ethical Committee of Urumqi General Hospital of Lanzhou Military Command of the Chinese People's Liberation Army.

Cell proliferation assays. The methyl thiazolyl tetrazolium (MTT) assay, which tests for cell proliferation and survival, was used in the present study, as previously described (14). Cells were incubated into 96 -well culture plate, $5 \times 10^{3}$ cells in each well, to incubate for $72 \mathrm{~h}$. In total, $50 \mu \mathrm{l}$ MTT solution was added to each well and incubated at $37^{\circ} \mathrm{C}$ for $4 \mathrm{~h}$. Following incubation, MTT was aspirated and $150 \mu \mathrm{l}$ dimethyl sulfoxide was added to each well to dissolve the formazan precipitate. Subsequently, an ELISA reader (SM600; Utrao, Shanghai, China) read the optical densities of the plates at $570 \mathrm{~nm}$.

Cell apoptosis assay. An Annexin V-Fluos staining kit (Roche-Boehringer) was used to detect early stages of apoptosis according with previous report (9). The cells were washed with phosphate-buffered saline (PBS) and stained according to the manufacturer's protocol. Slides were mounted with Permafluor mounting medium (Immunotech, Marseille, France) and viewed under a fluorescence microscope (Axiophot; Olympus, Tokyo, Japan).

RNA extraction. Total RNA was extracted from cells using TRIzol reagent (Invitrogen; Thermo Fisher Scientific, Inc.), according to the manufacturer's protocol. Reverse transcription-quantitative PCR (RT-qPCR) analyses for miRNAs were performed using TaqMan miRNA assays (Ambion; Thermo Fisher Scientific, Inc.) in an iQ5 Real-Time PCR Detection system (Bio-Rad Laboratories, Inc., Hercules, CA, USA). U6 small nuclear RNA was used as endogenous control for data normalization. Relative expression was calculated using the comparative threshold cycle $(\mathrm{Ct})$ method. To identify apoptotic nuclei, 4',6-diamidino-2-phenylindole (Roche, Mannheim, Germany) staining was performed according to the manufacturer's protocol.

Western blot. The cells were washed with ice-cold PBS and then lysed with protein lysate (Pierce, Rockford, IL, USA). After centrifugation at $5,000 \mathrm{x}$ f for $15 \mathrm{~min}$ at $4^{\circ} \mathrm{C}$, the protein concentration was measured with a bicinchoninic acid (BCA) protein assay kit (Pierce). Subsequently, $50 \mu \mathrm{g}$ aliquots of lysates were loaded on a $10 \%$ sodium dodecyl sulfate-polyacrylamide gel and transferred to a polyvinylidene difluoride membrane. The membranes were blocked with $5 \%$ skimmed dry milk in Tris-buffered saline ( $\mathrm{pH} 7.4$ ) containing $0.05 \%$ Tween 20 , and were incubated with Cox-2 antibodies (sc-514489; mouse monoclonal; 1:200 dilution; Santa Cruz Biotechnology, Inc., Dallas, TX, USA) and horseradish peroxidase-conjugated mouse secondary antibodies (sc-2383; 1:5,000 dilution; Santa Cruz Biotechnology, Inc.), according to the manufacturer's instructions. The protein of interest was visualized using an enhanced chemiluminescence western blotting substrate 
A

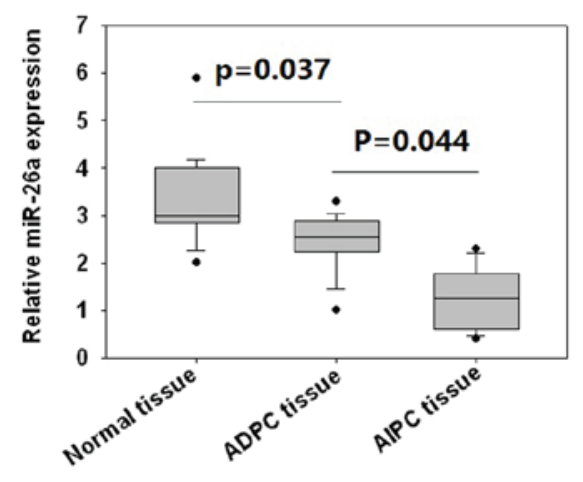

B

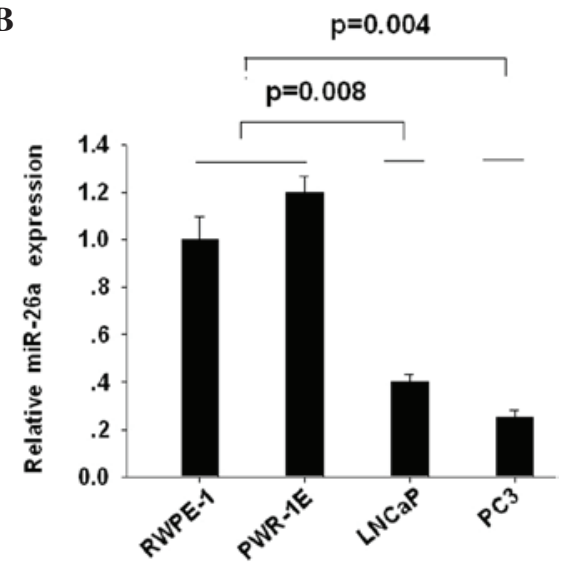

Figure 1. miR-26a expression is decreased in prostate cancer tissues and cells. (A) qPCR results comparing miR-26a levels between ADPC and AIPC tissues and normal tissues. (B) qPCR results comparing miR-26a levels among among normal prostate (RWPE-1 and PWR-1E), ADPC (LNCaP) and AIPC (PC3) cell lines. qPCR, quantitative polymerase chain reaction; ADPC, androgen-dependent prostate cancer; AIPC, androgen-independent prostate cancer; miR-26a, microRNA-26a.

(Pierce) and the Chemidoc XRS Gel Documentation system (Bio-Rad Laboratories, Inc.). Antibodies against glyceraldehyde 3-phosphate dehydrogenase (G8795; mouse monoclonal; 1:5,000 dilution) were obtained from Sigma-Aldrich (St. Louis, MO, USA).

Statistical analysis. The results are expressed as the mean \pm standard deviation of at least 3 separate experiments performed in triplicate. The differences between groups were determined using two-tailed Student's $t$-test, using SPSS software (version 22; IBM Corporation, Armonk, NY, USA). $\mathrm{P}<0.05$ was considered to indicate a statistically significant difference. $\chi^{2}$ test or Fisher's exact test was used to analyze the association between miR-26a expression and the clinicopathological features.

\section{Results}

Expression of miR-26a was lower in AIPC tissues compared with ADPC tissues. miR-26a expression was assessed in 12 AIPC tissues and 20 ADPC cases, together with the matched adjacent non-tumor prostate tissues. It was found that AIPC tissues showed the lowest miR-26a expression, followed by ADPC groups ( $\mathrm{P}=0.037$; Fig. $1 \mathrm{~A})$, and the non-tumor tissues showed significantly increased miR-26a expression compared with prostate cancer tissues $(\mathrm{P}=0.044)$. To confirm these clinical findings, the miR-26a expression using the ADPC LNCaP cell line, AIPC PC 3 cell line and the 2 normal prostate epithelial RWPE-1 and PWR-1E cell lines. The expression of miR-26a was lowest in the PC3 cells, followed by LNCaP cells $(\mathrm{P}=0.02)$. RWPE-1 and PWR-1E cells showed higher miR-26a expression levels (Fig. 1B). These data indicated that miR-26a expression was decreased in prostate cancer tissues and cell lines, with AIPC showing lower miR-26a expression compared with ADPC.

miR-26a negatively regulated prostate cancer cell proliferation by enhancing cell apoptosis. LNCaP and PC3 cells were then transfected with miR-26a mimics, inhibitors and control for $24 \mathrm{~h}$ and the efficiency of transfection was shown in Fig. 2A and B. miR-26a mimics significantly reduced the number of live cells $(\mathrm{P}=0.02)$, while miR-26a inhibitors had little effect (Fig. 2C). The same trend was also observed in PC3 cells (Fig. 2D). Subsequently, the cell apoptosis rates were determined using the Annexin V-Fluos staining kit $\mathrm{I}$ in the LNCaP (Fig. 2E) and PC3 (Fig. 2F) cells miR-26a mimics significantly enhanced the apoptosis rate $(\mathrm{P}=0.01)$, while miR-26a had the opposite effect $(\mathrm{P}=0.008$; Fig. $2 \mathrm{E})$. The same trend was observed in PC3 cells (Fig. 2F). These data indicated that miR-26a performs an important role in apoptosis development.

COX-2 is a miR-26a target gene in prostate cancer cells. To determine the potential role of miR-26a in mediating apoptosis, bioinformatic approaches identified potential miR-26a targets, and miRNA target identification quality was improved through the combined use of prediction programs. COX-2 was identified as a putative miR-26a target gene (Fig. 3A). To directly address whether miR-26a binds to the 3'-UTR of target mRNAs, luciferase reporter vectors that contained the putative miR-26a binding sites within 3'-UTR and the mutant 3'-UTR were generated. As shown in Fig. 3B, a marked reduction in luciferase activity in cells transfected with miR-26a mimics and luciferase report vectors was observed $(\mathrm{P}=0.02)$. By contrast, no change of luciferase activity was observed in cells transfected with the mutant 3'-UTR constructs.

miR-26a mediates COX-2 expression via mRNA degradation. Measurement of the mRNA and protein levels of COX-2 in LNCaP and PC3 cells transfected with miR-26a mimics or inhibitors revealed that overexpression (OV) of miR-26a resulted in the downregulation of the mRNA and the protein levels of COX-2 $(\mathrm{P}=0.02)$, while miR-26a inhibitors had the opposite effect on the target proteins (Fig. 3C and D). These data suggest that COX-2 is a potential target of miR-26a in prostate cancer cells and miR-26a mediates COX-2 expression via mRNA degradation.

miR-26a increased cell apoptosis by targeting COX-2 expression. COX-2 expression was associated with cancer 
Table I. Association between miR-26a expression and clinicopathological variables of patients with prostate cancer.

\begin{tabular}{|c|c|c|c|c|}
\hline \multirow[b]{2}{*}{ Variables } & \multirow[b]{2}{*}{ Cases } & \multicolumn{2}{|c|}{ miR-26a expression } & \multirow[b]{2}{*}{ P-value } \\
\hline & & Low & High & \\
\hline Gleason score & & & & 0.02 \\
\hline$\leq 7$ & 13 & 2 & 11 & \\
\hline$>7$ & 19 & 11 & 8 & \\
\hline T stage & & & & 0.04 \\
\hline $\mathrm{I}+\mathrm{II}$ & 11 & 5 & 6 & \\
\hline III+IV & 21 & 17 & 4 & \\
\hline PSA dependence & & & & 0.04 \\
\hline AIPC & 20 & 14 & 6 & \\
\hline ADPC & 12 & 5 & 7 & \\
\hline
\end{tabular}

Low, miR-26a/U6 <2.1; and high, miR-26a/U6 $\geq 2.1$. miR, microRNA; PSA, prostate specific antigen; AIPC, androgen-independent prostate cancer; ADPC, androgen-dependent prostate cancer.

A

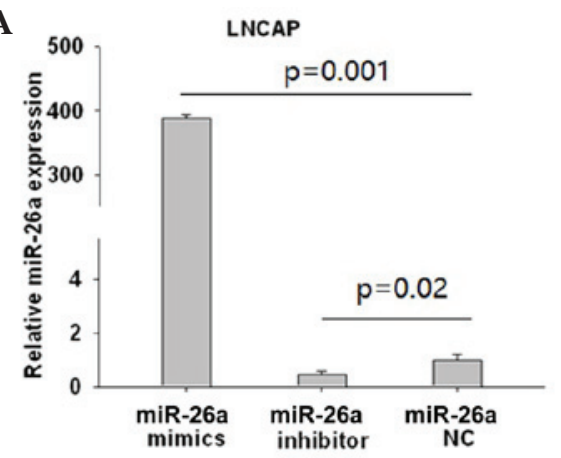

C

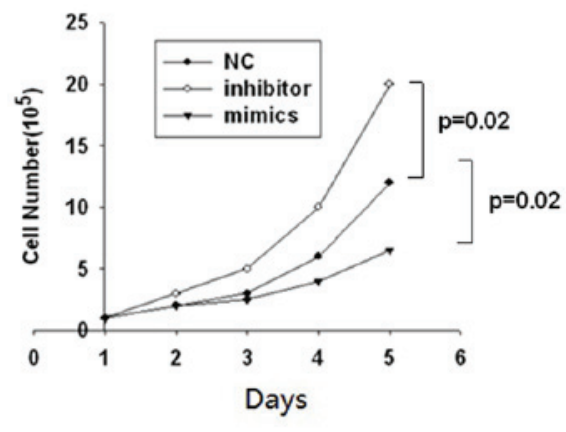

$\mathbf{E}$

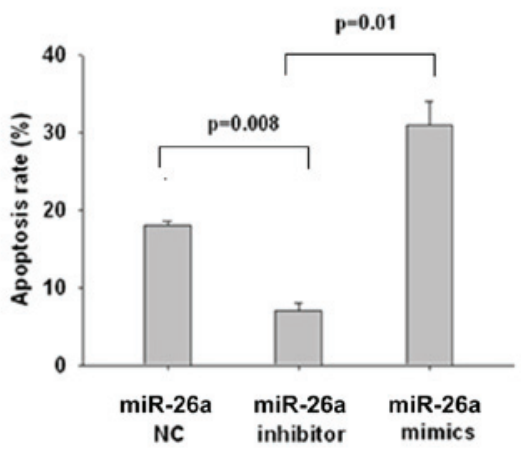

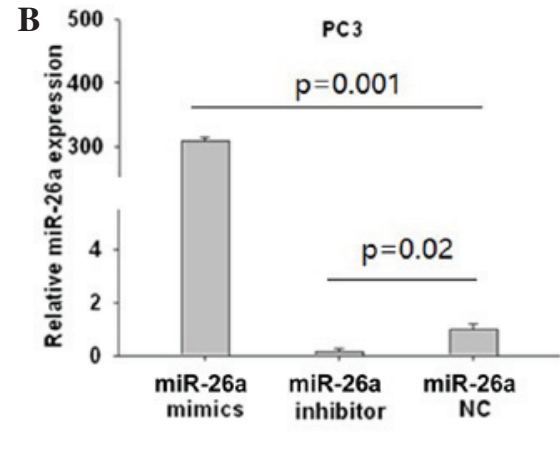

D

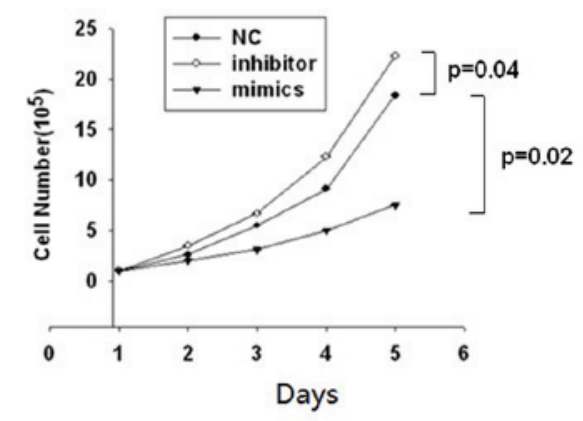

$\mathbf{F}$

$\mathrm{PC} 3$

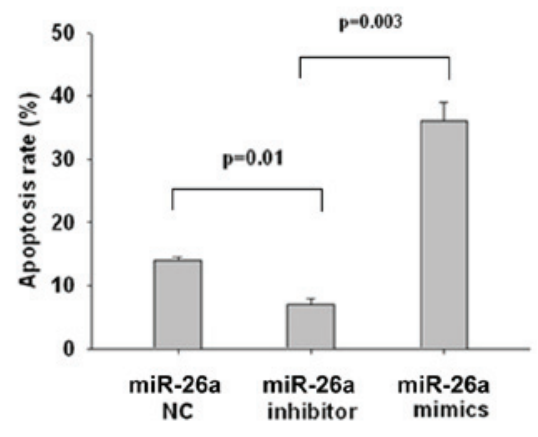

Figure 2. miR-26a negatively regulates prostate cancer cell proliferation by enhancing cell apoptosis. Cells were transfected with miR-26a mimics, inhibitor or NC. The relative miR-26a expression was calculated for (A) LNCaP and (B) PC3 cells. Subsequently, growth curves for (C) LNCaP and (D) PC3 cells were determined. Apoptosis rates for (E) LNCaP and (F) PC3 cells were also calculated. miR-26a, microRNA-26a; NC, negative control. 
A

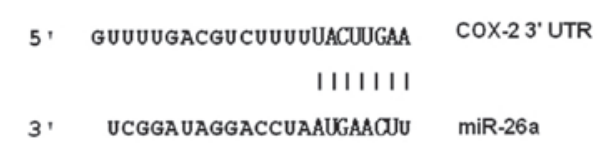

C

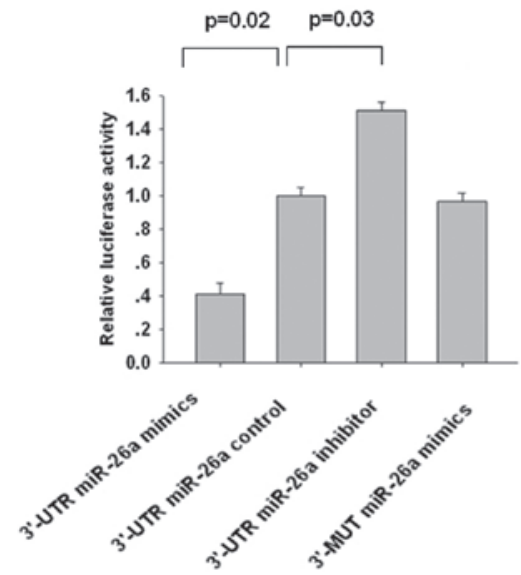

B

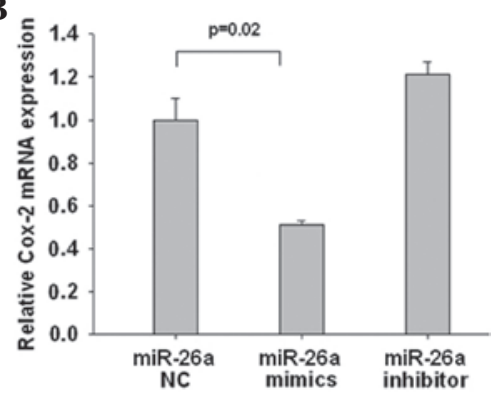

D cox-2

$$
\begin{aligned}
& \text { NC inhibitor mimic } \\
& \text { NC }
\end{aligned}
$$

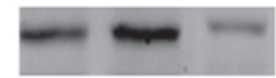

GAPDH

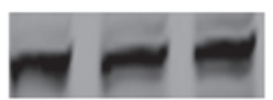

Figure 3. Identification of COX-2 as a potential target of miR-26a. (A) Region of the human COX-2 mRNA 3'-UTR predicted to be targeted by miR-26a (B) HEK-293 cells were transiently cotransfected with luciferase reporter vectors, and miR-26a mimics, inhibitor or NC. Luciferase activity was normalized to the activity of Renilla luciferase. (C and D) LNCaP cells were transfected with miR-26a mimics, inhibitor or NC. Subsequently, the (C) mRNA and (D) protein levels of COX-2 were determined. COX-2, cytochrome $c$ oxidase subunit II; 3'-UTR, 3'-untranslated region; NC, negative control; miR-26a, microRNA-26a; GAPDH, glyceraldehyde 3-phosphate dehydrogenase.

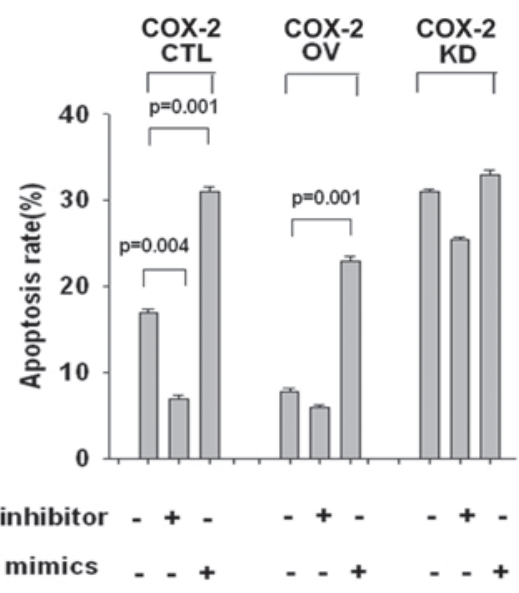

Figure 4. miR-26a enhanced cell apoptosis by suppressing COX-2 expression. LNCaP cells were transfected with the COX-2 OV plasmid, COX-2 KD plasmid or control vector, together with miR-26a mimics or inhibitor and then apoptosis rates were determined. COX-2, cytochrome $c$ oxidase subunit II; OV, overexpression; KD, knockdown; miR-26a, microRNA-26a.

cell apoptosis; therefore, the subsequent investigations were designed to explore whether miR-26a promotes apoptosis by suppressing COX-2. Cells were transiently transfected with the pcDNA3.1-COX-2 OV and siRNA for COX-2 knockdown (KD) plasmids, together with miR-26a mimics, control or inhibitor. As shown in Fig. 4, apoptosis of LNCaP cells was markedly reduced in the COX-2 OV group $(\mathrm{P}=0.001)$, and enhanced in the COX-2 KD group ( $\mathrm{P}=0.003)$, indicating that
COX-2 negatively regulated the development of apoptosis. Cotransfection with miR-26a mimics blocked the apoptosis reduction in the $\mathrm{OV}$ group $(\mathrm{P}=0.001)$, while in the $\mathrm{KD}$ group, miR-26a mimics and miR-26a inhibitor had little effect on the apoptosis rates. The same results were also observed in PC3 cells (data not shown). These data suggested that miR-26a upregulated apoptosis by suppressing COX-2 expression.

miR-26a expression and the association with clinical features. The association between miR-26a mRNA expression and clinical features of prostate cancer was analyzed in the high (miR-26a/U6 mean value $\geq 2.1$ ) and low (miR-26a/ U6 mean value $<2.1$ ) miR-26a expression groups, based on the results of RT-qPCR analysis. As shown in Table I, low miR-26a density was found in 14 cases of AIPC (70\%), and only in 5 cases of ADPC (41.7\%). In addition, intratumoral miR-26a density was negatively associated with the Gleason score $(\mathrm{P}=0.02)$ and $\mathrm{T}$ stage $(\mathrm{P}=0.04)$. These data indicated that patients with lower miR-26a density had an evidently poor prognosis (Table I).

\section{Discussion}

The expression level of miR-26a has been found to be downregulated in various types of cancers, and the miRNA was demonstrated to be a tumor suppressor that inhibits cancer cell proliferation by targeting various key proteins, such as EZH2 (15) and MCL-2 (16). By contrast, miR-26a was reported to facilitate carcinogenesis through the inhibition of 
phosphatase and tensin homolog (17). These studies indicated that miR-26 may play a different role in different tumors.

For prostate cancer, previous studies have demonstrated that miR-26a expression was lower in tumor tissues compared with their normal controls, which was also found in prostate cancer cells compared with normal cells (18). Consistent with these studies, the present study also found that the expression of miR-26a was downregulated in prostate cancer tissues and cell lines. Notably, miR-26a expression was significantly lower in the AIPC group compared with the ADPC group. Furthermore, a significant association was also observed between miR-26a expression and increased Gleason scores $(\mathrm{P}=0.02)$, and between miR-26a expression and tumor stage $(\mathrm{P}=0.04)$, indicating that the low miR-26a level was predictive of a poor prognosis.

Cell based assay indicated that miR-26a OV resulted in inhibition of tumor cell proliferation. In addition, the present study, to the best of our knowledge, is the first to demonstrate that negative regulatory function of miR-26a against COX-2 mediated apoptosis promotion was the main reason for tumor inhibition. COX-2 expression is suggested to be triggered by a variety of cytokines, and was shown to be a negative regulator of apoptosis (19). Accumulating evidence from experiments and clinical trials indicate that COX-2 plays a role in human carcinogenesis by inhibiting apoptosis, and COX-2 inhibitors were demonstrated to be able to arrest prostate cancer growth $(20,21)$. In the present study, apoptosis was markedly reduced in miR-26a inhibitor treatment, and then cotransfection si-COX-2 blocked this apoptosis reduction, suggesting that miR-26a-regulated cell apoptosis was dependent on COX-2.

Several miRNAs were demonstrated to modulate cell function through targeting COX-2. Wu et al (9) showed that miR-146a enhances Helicobacter pylori-induced cell apoptosis in human gastric cancer epithelial cells by targeting COX-2. Hao et al (22) demonstrated that enforced expression of miR-101 inhibits prostate cancer cell growth by modulating the COX-2 pathway. miR-16 has also been identified as a potential tumor regulator by targeting COX-2 (23). In addition to the present findings, these studies provide a novel cancer therapy based on miRNAs by directly inhibiting COX-2 expression.

The present study may assist in our understanding of the miR-26a regulation of AIPC formation in order to provide insight into the unique character of prostate cancer development. Furthermore, AIPC-related miR-26a could become a novel diagnostic or therapeutic tool in prostate cancer. The main limitation of the present study was that the small study and so further studies on a greater number of patients are ongoing to aid the generalizability of these conclusions.

\section{References}

1. Siegel RL, Miller KD and Jemal A: Cancer statistics, 2015. CA Cancer J Clin 65: 5-29, 2015.

2. Ren SC, Chen R and Sun YH: Prostate cancer research in China. Asian J Androl 15: 350-353, 2013.

3. Sooriakumaran P, Nyberg T, Akre O, Haendler L, Heus I, Olsson M, Carlsson S, Roobol MJ, Steineck G and Wiklund P: Comparative effectiveness of radical prostatectomy and radiotherapy in prostate cancer: Observational study of mortality outcomes. BMJ 348: g1502, 2014.

4. Kahn B, Collazo J and Kyprianou N: Androgen receptor as a driver of therapeutic resistance in advanced prostate cancer. Int J Biol Sci 10: 588-595, 2014.
5. Shi X, Gipp J, Dries M and Bushman W: Prostate progenitor cells proliferate in response to castration. Stem Cell Res 13: 154-163, 2014

6. Ianni M, Porcellini E, Carbone I, Potenzoni M, Pieri AM, Pastizzaro CD, Benecchi L and Licastro F: Genetic factors regulating inlammation and DNA methylation associated with prostate cancer. Prostate Cancer Prostatic Dis 16: 56-61, 2013.

7. Liu JB, Dai CM, Su XY, Cao L, Qin R and Kong QB: Gene microarray assessment of multiple genes and signal pathways involved in androgen-dependent prostate cancer becoming androgen independent. Asian Pac J Cancer Prev 15: 9791-9795, 2014.

8. Sullenger BA and Nair S: From the RNA world to the clinic. Science 352: 1417-1420, 2016.

9. Wu K, Yang L, Li C, Zhu CH, Wang X, Yao Y and Jia YJ: MicroRNA-146a enhances Helicobacter pylori induced cell apoptosis in human gastric cancer epithelial cells. Asian Pac J Cancer Prev 15: 5583-5586, 2014.

10. Zhao S, Ye X, Xiao L, Lian X, Feng Y, Li F and Li L: MiR-26a inhibits prostate cancer progression by repression of Wnt5a. Tumor Biol 35: 9725-97332014.

11. Shao Y, Li P, Zhu ST, Yue JP, Ji XJ, Ma D, Wang L, Wang YJ, Zong Y, Wu YD and Zhang ST: MiR-26a and miR-144 inhibit proliferation and metastasis of esophageal squamous cell cancer by inhibiting cyclooxygenase-2. Oncotarget 7 : 15173-15186, 2016.

12. Hickman OJ, Smith RA, Dasgupta P, Rao SN, Nayak S, Sreenivasan S, Vyakarnam A and Galustian C: Expression of two WFDC1/ps20 isoforms in prostate stromal cells induces paracrine apoptosis through regulation of PTGS2/COX-2. Br J Cancer 114: 1235-1242, 2016.

13. Jia RP, Xu LW, Su Q, Zhao JH, Li WC, Wang F and Xu Z: Cyclooxygenase-2 expression is dependent upon epidermal growth factor receptor expression or activation in androgen independent prostate cancer. Asian J Androl 10: 758-764, 2008.

14. Wang D, Zhu X, Cui C, Dong M, Jiang H, Li Z, Liu Z, Zhu W and Wang JG: Discovery of novel acetohydroxyacid synthase inhibitors as active agents against Mycobacterium tuberculosis by virtual screening and bioassay. J Chem Inf Model 53: 343-3532013.

15. Zhang B, Liu XX, He JR, Zhou CX, Guo M, He M, Li MF, Chen GQ and Zhao Q: Pathologically decreased miR-26a antagonizes apoptosis and facilitates carcinogenesis by targeting MTDH and EZH2 in breast cancer. Carcinogenesis 32: 2-9, 2011.

16. Gao J, Li L, Wu M, Liu M, Xie X, Guo J, Tang H and Xie X: MiR-26a inhibits proliferation and migration of breast cancer through repression of MCL-1. PLoS One 8: e65138, 2013.

17. Liu B, Wu X, Liu B, Wang C, Liu Y, Zhou Q and Xu K: MiR-26a enhances metastasis potential of lung cancer cells via AKT pathway by targeting PTEN. Biochim Biophys Acta 1822: 1692-1704, 2012.

18. Kato M, Goto Y, Matsushita R, Kurozumi A, Fukumoto I, Nishikawa R, Sakamoto S, Enokida H, Nakagawa M, Ichikawa T and Seki N: MicroRNA-26a/b directly regulate La-related protein 1 and inhibit cancer cell invasion in prostate cancer. Int J Oncol 47: 710-718, 2015.

19. Sun WH, Zhu F, Chen GS, Su H, Luo C, Zhao QS, Zhang Y, Shao Y, Sun J, Zhou SM, et al: Blockade of cholecystokinin-2 receptor and cyclooxygenase-2 synergistically induces cell apoptosis and inhibits the proliferation of human gastric cancer cells in vitro. Cancer Lett 263: 302-311, 2008.

20. Bieniek J, Childress C, Swatski MD and Yang W: COX-2 inhibitors arrest prostate cancer cell cycle progression by down-regulation of kinetochore/centromere proteins. Prostate 74: 999-1011, 2014.

21. Walther U, Emmrich K, Ramer R, Mittag N and Hinz B: Lovastatin lactone elicits human lung cancer cell apoptosis via a COX-2/PPAR $\gamma$-dependent pathway. Oncotarget 7: 10345$10362,2016$.

22. Hao Y, Gu X, Zhao Y, Greene S, Sha W, Smoot DT, Califano J, Wu TC and Pang X: Enforced expression of miR-101 inhibits prostate cancer cell growth by modulating the COX-2 pathway in vivo. Cancer Prev Res (Phila) 4: 1073-1083, 2011.

23. Young LE, Moore AE, Sokol L, Meisner-Kober N and Dixon DA: The mRNA stability factor HuR inhibits microRNA-16 targeting of COX-2. Mol Cancer Res 10: $167-180,2012$ 